\title{
QUASIORDERS, PRINCIPAL TOPOLOGIES, AND PARTIALLY ORDERED PARTITIONS
}

\author{
THOMAS A. RICHMOND \\ Department of Mathematics \\ Western Kentucky University \\ Bowling Green, Kentucky 42101
}

(Received August 26, 1996 and in revised form April 30, 1997)

\begin{abstract}
The quasiorders on a set $X$ are equivalent to the topologies on $X$ which are closed under arbitrary intersections. We consider the quaisorders on $X$ to be partial orders on the blocks of a partition of $X$ and use this approach to survey some fundamental results on the lattice of quasiorders on $X$.
\end{abstract}

KEY WORDS AND PHRASES: Quasiorder, principal topology, partially ordered partition, specialization topology, specialization order.

1991 AMS SUBJECT CLASSIFICATION CODES: 54A05, 54F05, 06 F99.

\section{INTRODUCTION}

The study of topology on finite sets can be framed entirely in terms of equivalence relations, partitions, and partial orders. A natural construction is to partially order the members (blocks) of a partition of an arbitrary (finite or infinite) set $X$. It is easy to see that such a partially ordered partition of $X$ is equivalent to a reflexive, transitive relation on $X$, that is, to a quasiorder on $X$. In 1937, Alexandroff showed that quasiorders on $X$ are equivalent to the topologies on $X$ in which arbitrary intersections of open sets are open. Such topologies are called principal topologies. For Hausdorff topological spaces, the associated quasiorder relation is equality. These connections between topology and discrete mathematics have not been as widely recognized as they deserve to be, largely due to the historical connections between topology and analysis, with the latter branch being primarily concerned with Hausdorff topological spaces. Non-Hausdorff topological spaces are finding numerous applications today in areas of computer science. (See the references in Kong et al. [14] and Kopperman [15].) Considering them as partially ordered partitions, we will study the principal topologies (quasiorders) on a set $X$, with particular attention to the lattice structure of the collection of all principal topologies on $X$. This collection is the collection of all topologies on $X$ if $X$ is finite.

A quasiorder (or preorder) on a set $X$ is a reflexive transitive relation on $X$. A partial order on $X$ is an antisymmetric quasiorder on $X$. A total order on $X$ is a partial order $\leq$ on $X$ in which every pair of elements $x, y \in X$ are comparable, that is, either $x \leq y$ or $y \leq x$. The discrete order on a set $X$ is $\Delta_{X}=\{(x, x): x \in X\}$. (Thus, the discrete order relation on $X$ is equality.) A symmetric quasiorder on $X$ is an equivalence relation on $X$. If $(X, \leq)$ is an (partially or quasi-) ordered set, the decreasing hull of a subset $B \subseteq X$ is defined to be $d_{X}(B)=\{x \in X: x \leq b$ for 
some $b \in B\}$. We say $B$ is a decreasing set in $X$ if $B=d_{X}(B)$. Increasing hulls and increasing sets are defined dually. A lattrce is a partially ordered set in which every pair of elements has a supremum and an infimum. A poset in which every nonempty subset has a supremum and an infimum is a complete lattice. If a lattice has a least element, it will be called the zero of the lattice, and denoted 0 . Similarly, if a lattice has a greatest element, it will be denoted 1. A partition of a set $X$ is a nonempty collection of mutually disjoint, nonempty subsets of $X$ whose union is $X$. The members of a partition $\mathcal{P}$ are called blocks of $\mathcal{P}$. There is a natural correspondence between an equivalence relation on $X$ and a partition of $X$ : The equivalence classes of an equivalence relation $R$ form the blocks of a partition $\mathcal{P}$, and a partition $\mathcal{P}$ determines an equivalence relation $R$ given by $x R y$ if and only if $x$ and $y$ are in the same block of $\mathcal{P}$. The equivalence class of $a \in X$ will be denoted by $[a]$. The complement of a set $A$ will be denoted $A^{c}$.

If $\mathcal{P}$ is a partition of a set $X$ and $\preceq$ is a partial order on $\mathcal{P}$, we call $(\mathcal{P}, \preceq)$ a partzally ordered partition, or popartition of $X$. For example, let $\mathcal{P}$ be the partition $\{[n, n+1): n$ is an integer $\}$ of the real line $\mathbf{R}$, and order $\mathcal{P}$ by agreeing that $[n, n+1) \preceq[m, m+1)$ if and only if $n \leq m$ in the usual order on $\mathbf{R}$. Then $(\mathcal{P}, \preceq)$ is a partially ordered partition of $\mathbf{R}$. (In fact, since $\preceq$ is a total order on $\mathcal{P},(\mathcal{P}, \preceq)$ is a totally ordered partition of $\mathbf{R}$.)

- Suppose $\leq$ is a quasiorder on $X$. There may exist distinct elements $a, b \in X$ with $a \leq b$ and $b \leq a$. Define a relation $R(\leq)$ on $X$ by $a R(\leq) b$ if and only if $a \leq b$ and $b \leq a$. It is easy to check that $R(\leq)$ is an equivalence relation on $X$. Let $\mathcal{P}$ be the corresponding partition. For blocks $A, B \in \mathcal{P}$, put $A \preceq B$ if and only if $a \leq b$ for some $a \in A$ and some $b \in B$. Equivalently, for blocks $[a],[b] \in \mathcal{P}$, put $[a] \preceq[b]$ if and only if there exist $a^{\prime} \in[a], b^{\prime} \in[b]$ with $a^{\prime} \leq b^{\prime}$. Now $\preceq$ is a partial order on the blocks of $\mathcal{P}$. In this manner, any quasiorder $\leq$ on $X$ generates a partially ordered partition $(\mathcal{P}, \preceq)$ of $X$. For example, the quasiorder $\sqsubseteq$ defined on $\mathbf{R}$ by $x \sqsubseteq y$ if and only if $\llbracket x \rrbracket \leq \llbracket y \rrbracket$ where $\llbracket x \rrbracket$ denotes the greatest integer less than or equal to $x$ in the usual order $\leq$ on $\mathbf{R}$, generates the totally ordered partition $(\mathcal{P}, \preceq)$ described in the last paragraph. On the other hand, if $(\mathcal{P}, \preceq)$ is a partially ordered partition of $X$, the relation $\leq$ defined on $X$ by $a \leq b$ if and only if $[a] \preceq[b]$ is a quasiorder on $X$. Thus, from any partially ordered partition of $X$, we can obtain a quasiorder on $X$, and from any quasiorder on $X$ we can obtain a partially ordered partition on $X$. Performing these two tricks successively would carry us from a popartition of $X$ to a popartition of $X$, or from a quasiorder on $X$ to a quasiorder on $X$. In either case, the original structure is identical to the final one. Thus, our notion of popartition is identical to the notion of quasiorder, with only a slight disguise.

In 1937, Alexandroff [2] noted another occurence of quasiorders in slight disguise. Quasiorders on a finite set are identical to the topologies on that set! A principal topology on a set $X$ is a topology on $X$ that is closed under arbitrary intersections of open sets. Observe that any finite topology, and in particular, any topology on a finite set, is a principal topology. Quasiorders on an arbitrary set are identical to the principal topologies on that set! If $\tau$ is a topology on $X$, the specralization order $\leq_{\tau}$ on $X$ is defined by $x \leq_{\tau} y$ if and only if $x \in \operatorname{cl}_{\tau}(\{y\})$, that is, if and only if every open neighborhood of $y$ contains $x$. It is easy to check that the specialization order $\leq_{\tau}$ is a quasiorder. If $\leq$ is a quasiorder on $X$, the set of all decreasing subsets of $X$ is a principal topology on $X$ called the specralization topology associated with $\leq$. Since complements of decreasing sets in a quasiordered set $(X, \leq)$ are increasing sets, the closure of a set $B \subseteq X$ relative to the specialization topology is the smallest increasing subset of $X$ that contains $B$. Thus, the increasing hull $i_{X}(B)$ of $B$ in $(X, \leq)$ is the closure of $B$ relative to the specialization topology.

A good account of principal topologies appears in Steiner [22]. Principal topologies are called 
Alexandroff discrete topologies or A-topologies in Erné and Stege [9]. Principal topologies are precisely the topologies in which every point has a smallest neighborhood. Karimpour [13] defines a complementary topology to be a topology in which the complement of every open set is open. Example 5 in Steen and Seebach [21] considers partition topologies, i.e., topologies on a set $X$ that have a base consisting of a partition of $X$. Complementary topologies and partition topologies are identical, and are examples of principal topologies.

A topology $\tau$ on a set $X$ is a $T_{0}$ topology if and only if for every pair of distinct points $x, y \in X$, there is a neighborhood of one of the points that excludes the other point. Equivalently, $\tau$ is a $T_{0}$ topology on $X$ if and only if for every pair of distinct points $x, y \in X$, one of the points is not in the closure of the other. If the topology $\tau$ is the specialization topology associated with a quasiorder $\leq$, the definition becomes $\tau$ is $T_{0}$ if and only if for every pair $x, y \in X$, either $x \notin i(y)$ or $y \notin i(x)$, i.e., either $x \geq y$ or $y \geq x$. Thus, we have that the specialization topology associated with a quasiorder $\leq$ is $T_{0}$ if and only if $\leq$ is antisymmetric, i.e., if and only if $\leq$ is a partial order. An excellent compilation of topological properties and the corresponding properties of the associated specialization quasiorder is given in Erné and Stege [9]. One can find there, for example, that a quasiorder on a finite set is an equivalence relation if and only if the specialization order is $T_{3}$.

\section{ELEMENTARY LATTICE PROPERTIES}

Given a set $X$, there are natural partial order relations on the set $Q(X)$ of all quasiorders on $X$, on the set $\operatorname{PoPar}(X)$ of all popartitions of $X$, and on the set $\operatorname{PrTop}(X)$ of all principal topologies on $X$. With their natural order, each set is a complete lattice, and the one-to-one correspondences between these sets discussed above are lattice homomorphisms. Below we will define these natural order relations, and examine the lattice stucture primarily by considering the lattice $\operatorname{PoPar}(X)$. Lattices of principal topologies and quasiorder lattices have been studied by Erné [8], Steiner [22], and Tuma [27]. Many of the works on principal topologies and quasiorders focus on applications to finite sets, in which case the lattice $\operatorname{Pr} \operatorname{Top}(X)$ of all principal topologies on $X$ is simply the lattice $\operatorname{Top}(X)$ of all topologies on $X$.

For topologies $\tau_{1}, \tau_{2} \in \operatorname{Top}(X)$, we say $\tau_{1} \leq_{T o p} \tau_{2}$ if and only if $\tau_{1} \subseteq \tau_{2}$ as subsets of the power set of $X$. (We would say $\tau_{1}$ is coarser than $\tau_{2}$, or $\tau_{2}$ is finer than $\tau_{1}$.) Now $\leq_{\text {Top }}$ is a partial order on $\operatorname{Top}(X)$, and $\left(\operatorname{Top}(X), \leq_{T o p}\right)$ is a complete lattice. $\left(\operatorname{Pr} \operatorname{Top}(X), \leq_{\text {Top }}\right)$ is also a complete lattice, though generally the supremum and infimum of a set in $\operatorname{Pr} \operatorname{Top}(X)$ may not agree with the supremum and infimum of the same set taken in $\operatorname{Top}(X)$. For quasiorders $\theta_{1}, \theta_{2} \in Q(X)$, we say $\theta_{1} \leq{ }_{Q} \theta_{2}$ if and only if $\theta_{1} \supseteq \theta_{2}$, i.e., if and only if the identity map $i d_{X}:\left(X, \theta_{2}\right) \longrightarrow\left(X, \theta_{1}\right)$ is increasing. (We use reverse inclusion here as the order on $Q(X)$ so that $\operatorname{Pr} \operatorname{Top}(X)$ and $Q(X)$ will be lattice isomorphic, as opposed to lattice anti-isomorphic.)

To describe the partial order $\leq$ on $\operatorname{PoPar}(X)$, we first consider the poset $\operatorname{Par}(X)$ of all partitions of $X$. For $\mathcal{P}, \mathcal{Q} \in \operatorname{Par}(X)$, we say $\mathcal{P} \leq \mathcal{Q}$ if and only if $[a]_{\mathcal{Q}} \subseteq[a]_{\mathcal{P}}$ for every $a \in X$, in which case we say $\mathcal{Q}$ is finer than $\mathcal{P}, \mathcal{Q}$ refines $\mathcal{P}$, or $\mathcal{P}$ is coarser than $\mathcal{Q}$. With this order, $\operatorname{Par}(X)$ is a complete lattice (Theorem I.5 of Ore [18]). Define a relation $\leq$ on $\operatorname{PoPar}(X)$ by $\left(\mathcal{P}, \preceq_{\mathcal{P}}\right) \leq\left(\mathcal{Q}, \preceq_{\mathcal{Q}}\right)$ if and only if $\mathcal{P} \leq \mathcal{Q}$ in $\operatorname{Par}(X)$ and the quotient function $f_{\mathcal{Q}, \mathcal{P}}:\left(\mathcal{Q}, \preceq_{\mathcal{Q}}\right) \longrightarrow$ $\left(\mathcal{P}, \preceq_{\mathcal{P}}\right)$ defined by $f\left([a]_{\mathcal{Q}}\right)=[a]_{\mathcal{P}}$ is increasing.

PROPOSITION 2.1. $\leq$ is a partial order on $\operatorname{PoPar}(X)$.

PROOF. Antisymmetry: If $\left(\mathcal{P}, \preceq_{\mathcal{P}}\right) \leq\left(\mathcal{Q}, \preceq_{\mathcal{Q}}\right)$ and $\left(\mathcal{Q}, \preceq_{\mathcal{Q}}\right) \leq\left(\mathcal{P}, \preceq_{\mathcal{P}}\right)$, then as members of $\operatorname{Par}(X), \mathcal{P}=\mathcal{Q}$, and since the quotient functions $f_{\mathcal{P}, \mathcal{Q}}$ and $f_{\mathcal{Q}, \mathcal{P}}$ are both increasing, the order on $\mathcal{P}$ must agree with the order on $\mathcal{Q}$, so $\left(\mathcal{P}, \preceq_{\mathcal{P}}\right)=\left(\mathcal{Q}, \preceq_{\mathcal{Q}}\right)$ as members of $\operatorname{PoPar}(X)$. Transitivity: 
If $\left(\mathcal{P}, \preceq_{\mathcal{P}}\right) \leq\left(\mathcal{Q}, \preceq_{\mathcal{Q}}\right)$ and $\left(\mathcal{Q}, \preceq_{\mathcal{Q}}\right) \leq\left(\mathcal{R}, \preceq_{\mathcal{R}}\right)$, then $\mathcal{P} \leq \mathcal{R}$ in $\operatorname{Par}(X)$, and as the composition of two increasing functions, $f_{\mathcal{P}, \mathcal{R}}=f_{\mathcal{Q}, \mathcal{R}} \circ f_{\mathcal{P}, \mathcal{Q}}$ is increasing. Reflexivity: $\mathcal{P} \leq \mathcal{P}$ in $\operatorname{Par}(X)$ and the identity map is increasing.

PROPOSITION 2.2. If $\left(\left(\mathcal{P}_{\alpha}, \preceq_{\alpha}\right)\right)_{\alpha \in A}$ is a collection of popartitions of $X$, then $\bigvee_{\alpha \in A}\left(\mathcal{P}_{\alpha}, \preceq_{\alpha}\right)$ in $\operatorname{PoPar}(X)$ exists; its underlying partition is $\mathcal{P}=\bigvee_{\alpha \in A} \mathcal{P}_{\alpha}$ (supremum in $\operatorname{Par}(X)$ ) and its order $\leq_{\mathcal{P}}$ is given by $[a]_{\mathcal{P}} \leq_{\mathcal{P}}[b]_{\mathcal{P}}$ if and only if $[a]_{\mathcal{P}_{\alpha}} \leq_{\alpha}[b]_{\mathcal{P}_{\alpha}}$ for every $\alpha \in A$.

PROOF. It is easy to verify that $\leq_{\mathcal{P}}$ is a partial order. With $\mathcal{P}$ as described, $\mathcal{P}_{\alpha} \leq \mathcal{P}$ and $f_{\mathcal{P}, \mathcal{P}_{\alpha}}$ is clearly increasing for any $\alpha \in A$, so $\left(\mathcal{P}, \preceq_{\mathcal{P}}\right)$ is an upper bound of $\left(\left(\mathcal{P}_{\alpha}, \preceq_{\alpha}\right)\right)_{\alpha \in A}$. Suppose $\left(\mathcal{Q}, \preceq_{\mathcal{Q}}\right)$ is also an upper bound of $\left(\left(\mathcal{P}_{\alpha}, \preceq_{\alpha}\right)\right)_{\alpha \in A}$. Then as elements of $\operatorname{Par}(X), \mathcal{P} \leq \mathcal{Q}$. To see $\left(\mathcal{P}, \preceq_{\mathcal{P}}\right)$ is the least upper bound of $\left(\left(\mathcal{P}_{\alpha}, \preceq_{\alpha}\right)\right)_{\alpha \in A}$, it only remains to show that $f_{\mathcal{Q}, \mathcal{P}}$ is increasing. Suppose $[a]_{\mathcal{Q}} \leq_{\mathcal{Q}}[b]_{\mathcal{Q}}$. Since $\left(\mathcal{Q}, \preceq_{\mathcal{Q}}\right) \geq\left(\mathcal{P}_{\alpha}, \preceq_{\alpha}\right)$ for every $\alpha \in A$, we have $[a]_{\mathcal{P}_{\alpha}} \leq_{\alpha}[b]_{\mathcal{P}_{\alpha}}$ for every $\alpha \in A$, and thus $[a]_{\mathcal{P}} \leq_{\mathcal{P}}[b]_{\mathcal{P}}$.

A poset in which every nonempty subset has a supremum is called a $V$-complete semilattrce. Any V-complete semilattice $P$ with a least element is a complete lattice, for it is easily shown that for any $S \subseteq P$, the supremum of the set of lower bounds of $S$ is itself a lower bound of $S$ and is thus the infimum of $S$. The dual result for $\wedge$-complete semilattices holds as well. By Proposition 2.2, $\operatorname{PoPar}(X)$ is a $\mathrm{V}$-complete semilattice. It has a smallest element, namely the indiscrete partition $\{X\}$. This proves the following results.

COROLLARY 2.3. $\quad \operatorname{PoPar}(X)$ is a complete lattice.

A description of the infimum of a set $\left(\left(\mathcal{P}_{\alpha}, \preceq_{\alpha}\right)\right)_{\alpha \in A}$ in $\operatorname{PoPar}(X)$ will be useful. In $\operatorname{Par}(X)$, $\bigwedge_{\alpha \in A} \mathcal{P}_{\alpha}$ can be described (see p. 579 in Ore [18]) as the partition of $X$ whose blocks are the maximal chain connected subfamilies of $\bigcup_{\alpha \in A} \mathcal{P}_{\alpha}$ where a family $\mathcal{F}$ is chain connected if for any $A, B \in \mathcal{F}$, there exist $A_{\imath} \in \mathcal{F}(i=1, \ldots, n)$ with $A \cap A_{\imath} \neq \emptyset, A_{\imath} \cap A_{\imath+1} \neq \emptyset$, and $A_{n} \cap B \neq \emptyset$. Viewing the partitions $\left(\mathcal{P}_{\alpha}\right)_{\alpha \in A}$ as equivalence relations $\sim_{\alpha}, \wedge_{\alpha \in A} \mathcal{P}_{\alpha}$ can be described by the relation $a \sim b$ if and only if there exists a chain of equivalences $a \sim_{\alpha_{0}} a_{1}, a_{2} \sim_{\alpha_{1}} a_{\imath+1}, a_{n} \sim_{\alpha_{n}} b$ for some $\alpha_{\imath} \in A(i=0,1, \ldots, n)$. We will say $a, b \in X$ are loop connected in $\bigcup_{\alpha \in A} \mathcal{P}_{\alpha}$ if there exist $\alpha_{\imath} \in A$ and $a_{\imath} \in X \quad(i=0,1, \ldots, n)$ with $a_{0}=a_{n}=a$, and $b=a_{\imath}$ for some $i$ and

$$
\left[a_{\imath}\right]_{\mathcal{P}_{\alpha_{2}}} \leq_{\alpha_{2}}\left[a_{\imath+1}\right]_{\mathcal{P}_{\alpha_{i}}} \text { for } i=0,1, \ldots, n-1 \text {. }
$$

That is, $a, b \in X$ are loop connected in $\bigcup_{\alpha \in A} \mathcal{P}_{\alpha}$ if there is an increasing loop from $[a]_{\mathcal{P}_{\alpha_{0}}}$ to $[a]_{\mathcal{P}_{\alpha_{n}}}$ which passes through $[b]_{\mathcal{P}_{\alpha_{i}}}$ for some $i$. Note that if $a$ is chain connected to $\dot{b}$, then the chain from $a$ to $b$ followed by the chain from $b$ to $a$ shows that $a$ and $b$ are loop connected simply by taking $\leq_{\alpha_{i}}$ in (2.1) to be equality at each link. "Is loop connected to" is an equivalence relation $\mathcal{P}$ on $X$. Define $\preceq$ on $\mathcal{P}$ by $[a]_{\mathcal{P}} \preceq[b]_{\mathcal{P}}$ if and only if there exists a chain as in (2.1) with $a_{0}=a$ and $a_{n}=b$. This defines a partial order on $\mathcal{P}$. Since chain connectedness implies loop connectedness, $\mathcal{P}$ is coarser than $\bigwedge_{\alpha \in A} \mathcal{P}_{\alpha}$ where the infimum is taken in $\operatorname{Par}(X)$. From the construction of $(\mathcal{P}, \preceq), \mathcal{P}$ is the finest partition below each $\mathcal{P}_{\alpha}$, and $\mathcal{P}$ was given the minimal order necessary to make it a lower bound of $\left(\left(\mathcal{P}_{\alpha}, \preceq_{\alpha}\right)\right)_{\alpha \in A}$. Thus, in $\operatorname{PoPar}(X),(\mathcal{P}, \preceq)=\bigwedge_{\alpha \in A}\left(\left(\mathcal{P}_{\alpha}, \preceq_{\alpha}\right)\right)$.

Note that the partition underlying $\bigwedge_{\alpha \in A}\left(\left(\mathcal{P}_{\alpha}, \preceq_{\alpha}\right)\right.$ ) (infimum in $\left.\operatorname{PoPar}(X)\right)$ is not generally the same as $\bigwedge_{\alpha \in A} \mathcal{P}_{\alpha}$ (infimum in $\left.\operatorname{Par}(X)\right)$. For example, if $\left(\mathcal{P}_{1}, \prec_{1}\right)=\left(\left\{A, A^{c}\right\}, A \prec_{1} A^{c}\right)$ and $\left(\mathcal{P}_{2}, \prec_{2}\right)=\left(\left\{A, A^{c}\right\}, A^{c} \prec_{2} A\right)$ are popartitions of $X$, the infimum of their underlying partitions in $\operatorname{Par}(X)$ is $\left\{A, A^{c}\right\}$, while their infimum in $\operatorname{PoPar}(X)$ is $(\{X\}, \Delta)$. However, from the description of suprema and infima in $\operatorname{PoPar}(X)$, we see that for discretely ordered popartitions, the partitions underlying suprema and infima in $\operatorname{PoPar}(X)$ agree with the suprema and infima of the underlying partitions in $\operatorname{Par}(X)$. Thus we have the following result. 
PROPOSITION 2.4. $\quad \operatorname{Par}(X)$ is a sublattice of $\operatorname{PoPar}(X)$.

It is shown in Pudlák and Tơma [19] that "every finite lattice can be embedded in a finite partition lattice". With Proposition 2.4, this implies that every finite lattice can be embedded in $\operatorname{PoPar}(X)$ for some finite set $X$.

\section{ATOMS AND COVERINGS IN PoPar(X)}

We say $b$ covers $a$ in a poset $X$ if $a<b$, and $a \leq c \leq b$ implies $c \in\{a, b\}$. If $X$ is a lattice with smallest element 0 , an atom is an element that covers 0 . If $X$ has a largest element 1 , a coatom is an element covered by 1 . The smallest and largest elements of $\operatorname{PoPar}(X)$ are the indiscrete partition $\{X\}$ and the discretely ordered discrete partition $(\{\{x\}: x \in X\}, \Delta)$, respectively. The atoms of $\operatorname{PoPar}(X)$ are the two element chains in $\operatorname{PoPar}(X)$, that is, the partitions of form $\left\{A, A^{c}\right\}$ with $A \prec A^{c}$ or $A^{c} \prec A$. The coatoms are the discrete partitions with orders of the form $\Delta_{X} \cup\left\{\left(x_{0}, x_{1}\right)\right\}$ where $x_{0}$ and $x_{1}$ are distinct elements of $X$. Every nonzero element of $\operatorname{PoPar}(X)$ is above an atom: For $(\mathcal{P}, \preceq) \in \operatorname{PoPar}(X)$, let $A \in \mathcal{P}$ and consider $d_{\mathcal{P}}(A)=\{B \in \mathcal{P}: B \preceq A$ in $(\mathcal{P}, \preceq)\}$. Now if $i_{\mathcal{P}}(A)$ is defined dually, $d_{\mathcal{P}}(A)=i_{\mathcal{P}}(A)=\mathcal{P}$ would imply $A$ is maximum and minimum in $(\mathcal{P}, \preceq)$, so $(\mathcal{P}, \preceq)=(\{A\}, \Rightarrow)$ would be the zero element of $\operatorname{PoPar}(X)$. If $(\mathcal{P}, \preceq) \neq 0$, assume, without loss of generality, that $d_{\mathcal{P}}(A) \neq \mathcal{P}$ so that $d_{\mathcal{P}}(A)$ and $\mathcal{P} \backslash d_{\mathcal{P}}(A)$ are disjoint nonempty sets, the former decreasing and the latter increasing, that partition $\mathcal{P}$, and therefore partition $X$. $(\mathcal{P}, \preceq)$ is above the atom $\mathcal{P}_{A} \equiv\left(\left\{d_{\mathcal{P}}(A), \mathcal{P} \backslash d_{\mathcal{P}}(A)\right\}, \quad d_{\mathcal{P}}(A)<\mathcal{P} \backslash d_{\mathcal{P}}(A)\right)$. Indeed, every nonzero popartition is the supremum of the atoms below it: If $(\mathcal{P}, \preceq) \in \operatorname{PoPar}(X)$ and $A \in \mathcal{P}$ is not maximum, $\mathcal{P}_{A}$ gives an atom below $(\mathcal{P}, \preceq)$, and if $A$ is not minimum, $\left(\left\{i_{\mathcal{P}}(A), \mathcal{P} \backslash i_{\mathcal{P}}(A)\right\}, \quad i_{\mathcal{P}}(A)>\mathcal{P} \backslash i_{\mathcal{P}}(A)\right) \equiv \mathcal{P}^{A}$ gives an atom below $(\mathcal{P}, \preceq)$. We claim $\vee\left(\left\{\mathcal{P}_{A}: A \in \mathcal{P}, A\right.\right.$ not maximum in $\left.\mathcal{P}\right\} \vee\left\{\mathcal{P}^{\mathcal{A}}: \mathcal{A} \in \mathcal{P}, \mathcal{A}\right.$ not minimum in $\mathcal{P}\})=(\mathcal{P}, \preceq)$. The atoms involved in the expression above only partition $X$ into partitions of the blocks of $\mathcal{P}$, so the supremum of these atoms will give a partition coarser than $\mathcal{P}$. However, since $\mathcal{P}^{A} \vee \mathcal{P}_{A}$ (or simply the one that exists, if both do not exist) gives a partition of $X$ with $A$ as a block, we see that the partition underlying the supremum of atoms given above is simply $\mathcal{P}$. Suppose $[b]_{\mathcal{P}}$ and $[c]_{\mathcal{P}}$ are blocks in $\mathcal{P}$ with $[b]_{\mathcal{P}} \leq[c]_{\mathcal{P}}$. Each atom included in the supremum above partitions $\mathcal{P}$ into an increasing set $I$ and a decreasing set $D$. If $[b]_{\mathcal{P}} \in I$, then $[c]_{\mathcal{P}} \in I$ so that $[b]=[c]$ in any of the atoms listed. Similarly, $[b]=[c]$ if $[c]_{\mathcal{P}} \in D$. In the remaining case, $[b]_{\mathcal{P}} \in D,[c]_{\mathcal{P}} \in I$, since $D<I$ in each atom, we have $[b] \leq[c]$ in each atom. Conversely, if $[b]_{\mathcal{P}} \mathbb{Z}[c]_{\mathcal{P}}$, then for $B=[b]_{\mathcal{P}}$ we have $[b]_{\mathcal{P}_{B}} \mathbb{Z}[c]_{\mathcal{P}_{B}}$. Thus, the order on the supremum of atoms given above does agree with the order $\preceq$ on $\mathcal{P}$.

Before investigating coverings in $\operatorname{PoPar}(X)$, observe that $\mathcal{P}$ covers $\mathcal{Q}$ in $\operatorname{Par}(X)$ if and only if $\mathcal{Q}$ is obtained from $\mathcal{P}$ by combining two blocks of $\mathcal{P}$. For coverings in the collection $P o(X)$ of all partial orders on $X$ ordered by reverse inclusion (i.e., $\left(X, \leq_{1}\right) \leq\left(X, \leq_{2}\right)$ if and only if the identity function id: $\left(X, \leq_{1}\right) \longrightarrow\left(X, \leq_{2}\right)$ is increasing), we have the following result.

LEMMA 3.1. $\quad \theta$ covers $\psi$ in $P o(X)$ if and only if $\psi=\theta \cup\{(a, b)\}$ for some pair $(a, b) \in X^{2} \backslash \theta$.

PROOF. Suppose $\theta$ covers $\psi$. Then $\theta \subset \psi$. Let $(a, b) \in \psi \backslash \theta$. Now $\phi=\theta \cup\left\{(x, y): x \leq_{\theta}\right.$ $\left.a, b \leq_{\theta} y\right\}$ is a partial order with $\theta \subset \phi \subseteq \psi$. Since $\theta$ covers $\psi$, we have $\phi=\psi$, and thus, if there exists another pair $(c, d) \in \psi \backslash \theta,(c, d) \neq(a, b)$, then $c \leq_{\theta} a$ and $b \leq_{\theta} d$, and at least one of these inequalitites is strict. Now $\phi^{\prime}=\theta \cup\left\{(x, y): x \leq_{\theta} c, d \leq_{\theta} y\right\}$ is a partial order with $\theta \subset \phi^{\prime} \subset \phi=\psi$, contrary to the fact that $\theta$ covers $\psi$. Thus, if $\theta \operatorname{covers} \psi$, then $\theta \subset \psi$ and $|\psi \backslash \theta|=1$. The converse is trivial.

Since only the covering relations are depicted in the Hasse diagram for a poset, a character- 
ization of the covering relations in $\operatorname{PoPar}(X)$ would be a useful tool. Although the following result does not characterize the covering relations, it narrows down the search for coverings and is still very helpful in understanding and describing the lattice $\operatorname{PoPar}(X) \approx \operatorname{PrTop}(X) \approx Q(X)$, particularly when $X$ is a small finite set, as we will see in the next section.

PROPOSITION 3.2. If $\left(\mathcal{P}, \preceq_{\mathcal{P}}\right)$ covers $\left(\mathcal{Q}, \preceq_{\mathcal{Q}}\right)$ in $\operatorname{PoPar}(X)$, then one of the following conditions holds:

1. $\mathcal{P}=\mathcal{Q}$ and $\preceq_{\mathcal{Q}}$ contains exactly one more ordered pair than $\preceq_{\mathcal{p}}$.

2. $\left(\mathcal{Q}, \preceq_{\mathcal{Q}}\right)$ is obtained by identifying a pair of blocks from $\left(\mathcal{P}, \preceq_{\mathcal{P}}\right)$, one of which covers the other in $\left(\mathcal{P}, \preceq_{\mathcal{P}}\right)$.

PROOF. Suppose $\left(\mathcal{P}, \preceq_{\mathcal{P}}\right)$ covers $\left(\mathcal{Q}, \preceq_{\mathcal{Q}}\right)$ in $\operatorname{PoPar}(X)$. If $\mathcal{P}=\mathcal{Q}$, then $\preceq_{\mathcal{P}}$ covers $\preceq_{\mathcal{Q}}$ in $P_{o}(B)$ where $B$ is the set of blocks in $\mathcal{P}=\mathcal{Q}$, and the lemma above implies that $\preceq_{\mathcal{Q}}$ contains exactly one more ordered pair than $\preceq_{\mathcal{P}}$. Now suppose $\mathcal{P} \neq \mathcal{Q}$, that is, $\mathcal{Q}<\mathcal{P}$. Suppose that $\mathcal{P}$ does not cover $\mathcal{Q}$ in $\operatorname{Par}(X)$. Then there exist distinct blocks $[x]_{\mathcal{P}},[y]_{\mathcal{P}},[z]_{\mathcal{P}}$ in $\mathcal{P}$ with $[x]_{\mathcal{P}} \neq[x]_{\mathcal{Q}},[y]_{\mathcal{P}} \neq[y]_{\mathcal{Q}}$, and $[z]_{\mathcal{P}} \neq[z]_{\mathcal{Q}}$. Without loss of generality, $[x]_{\mathcal{Q}}=[y]_{\mathcal{Q}}$ and $[x]_{\mathcal{P}} \nprec_{\mathcal{P}}[y]_{\mathcal{P}}$, so that $\left(\mathcal{R}, \preceq_{\mathcal{R}}\right)=\left\{i_{\mathcal{P}}\left([x]_{\mathcal{P}}\right), X \backslash i_{\mathcal{P}}\left([x]_{\mathcal{P}}\right)\right\}$ with $i_{\mathcal{P}}\left([x]_{\mathcal{P}}\right)$ as greatest element is an atom below $\left(\mathcal{P}, \preceq_{\mathcal{P}}\right)$ in $\operatorname{PoPar}(X)$ in which $[x]_{\mathcal{R}} \neq[y]_{\mathcal{R}}$. If the blocks of $\mathcal{R}$ do not split $[z]_{\mathcal{Q}}$, i.e., if $[z]_{\mathcal{Q}} \subseteq[z]_{\mathcal{R}}$, then in $\left(\mathcal{S}, \preceq_{\mathcal{S}}\right)=\sup \left\{\left(\mathcal{Q}, \preceq_{\mathcal{Q}}\right),\left(\mathcal{R}, \preceq_{\mathcal{R}}\right)\right\}$ we have $[x]_{\mathcal{S}} \neq[y]_{\mathcal{S}}$ even though $[x]_{\mathcal{Q}}=[y]_{\mathcal{Q}}$, and $[z]_{\mathcal{S}}=[z]_{\mathcal{Q}} \neq[z]_{\mathcal{P}}$, so that $\left(\mathcal{S}, \preceq_{\mathcal{S}}\right)$ is strictly between $\left(\mathcal{P}, \preceq_{\mathcal{P}}\right)$ and $\left(\mathcal{Q}, \preceq_{\mathcal{Q}}\right)$ in $\operatorname{PoPar}(X)$, contrary to $\left(\mathcal{P}, \preceq_{\mathcal{P}}\right)$ covering $\left(\mathcal{Q}, \preceq_{\mathcal{Q}}\right)$. If the blocks of $\mathcal{R}$ do split $[z]_{\mathcal{Q}}$, i.e., if there exist $z^{\prime}, z^{\prime \prime} \in[z]_{\mathcal{Q}}$ with $[x]_{\mathcal{P}} \preceq_{\mathcal{P}}\left[z^{\prime}\right]_{\mathcal{P}}$ and $[x]_{\mathcal{P}} \swarrow_{\mathcal{P}}\left[z^{\prime \prime}\right]_{\mathcal{P}}$, then $\left(\mathcal{R}^{\prime}, \preceq_{\mathcal{R}}^{\prime}\right)=\left\{i_{\mathcal{P}}\left(\left[z^{\prime}\right]_{\mathcal{P}}\right), X \backslash \imath_{\mathcal{P}}\left(\left[z^{\prime}\right]_{\mathcal{P}}\right)\right\}$ with $i_{\mathcal{P}}\left(\left[z^{\prime}\right]_{\mathcal{P}}\right)$ as greatest element is an atom below $\left(\mathcal{P}, \preceq_{\mathcal{P}}\right)$ in $\operatorname{PoPar}(X)$ which splits $[z]_{\mathcal{Q}}$. Since $[x]_{\mathcal{P}} \prec_{\mathcal{P}}\left[z^{\prime}\right]_{\mathcal{P}}$ and $[x]_{\mathcal{P}} \nprec_{\mathcal{P}}[y]_{\mathcal{P}}$, it follows that $[x]_{\mathcal{P}},[y]_{\mathcal{P}} \in X \backslash i_{\mathcal{P}}\left(\left[z^{\prime}\right]_{\mathcal{P}}\right)$, and thus $[x]_{\mathcal{R}^{\prime}}=[y]_{\mathcal{R}^{\prime}}$. Now in $\left(\mathcal{S}^{\prime}, \preceq_{\mathcal{S}}^{\prime}\right)=\sup \left\{\left(\mathcal{Q}, \preceq_{\mathcal{Q}}\right),\left(\mathcal{R}^{\prime}, \preceq_{\mathcal{R}}^{\prime}\right)\right\}$ we have $[x]_{\mathcal{S}^{\prime}}=[y]_{\mathcal{S}^{\prime}}$ even though $[x]_{\mathcal{P}} \neq[y]_{\mathcal{P}}$, and $\left[z^{\prime}\right]_{\mathcal{S}^{\prime}} \neq\left[z^{\prime \prime}\right]_{\mathcal{S}^{\prime}}$ even though $\left[z^{\prime}\right]_{\mathcal{Q}}=\left[z^{\prime \prime}\right]_{\mathcal{Q}}=[z]_{\mathcal{Q}}$. Thus, $\left(\mathcal{S}^{\prime}, \preceq_{\mathcal{S}}^{\prime}\right)^{\prime}$ is strictly between $\left(\mathcal{P}, \preceq_{\mathcal{P}}\right)$ and $\left(\mathcal{Q}, \preceq_{\mathcal{Q}}\right)$ in $\operatorname{PoPar}(X)$, contrary to $\left(\mathcal{P}, \preceq_{\mathcal{P}}\right)$ covering $\left(\mathcal{Q}, \preceq_{\mathcal{Q}}\right)$. Thus, if $\left(\mathcal{P}, \preceq_{\mathcal{P}}\right)$ covers $\left(\mathcal{Q}, \preceq_{\mathcal{Q}}\right)$ in $\operatorname{PoPar}(X)$ and $\mathcal{P} \neq \mathcal{Q}$, there can not exist three distinct blocks $[x]_{\mathcal{P}},[y]_{\mathcal{P}},[z]_{\mathcal{P}}$ in $\mathcal{P}$ with $[x]_{\mathcal{P}} \neq[x]_{\mathcal{Q}},[y]_{\mathcal{P}} \neq[y]_{\mathcal{Q}}$, and $[z]_{\mathcal{P}} \neq[z]_{\mathcal{Q}}$. Hence the partition $\mathcal{Q}$ is obtained from $\mathcal{P}$ by identifying two blocks $A, B$ of $\mathcal{P}$, i.e., $\mathcal{P}$ covers $\mathcal{Q}$ in $\operatorname{Par}(X)$. Finally, we show that $A$ and $B$ are related in $\left(\mathcal{P}, \preceq_{\mathcal{P}}\right)$. If they are not related, let $\left(\mathcal{R}, \preceq_{\mathcal{R}}\right)$ be the popartition with $\mathcal{R}=\mathcal{P}$ and with $\theta_{\mathcal{R}}=\theta_{\mathcal{P}} \cup\left\{\{C, D\}: C \preceq_{\mathcal{P}} A, B \preceq_{\mathcal{P}} D\right\}$. Now $\left(\mathcal{R}, \preceq_{\mathcal{R}}\right)$ is strictly between $\left(\mathcal{P}, \preceq_{\mathcal{P}}\right)$ and $\left(\mathcal{Q}, \preceq_{\mathcal{Q}}\right)$ in $\operatorname{PoPar}(X)$, again contradicting that $\left(\mathcal{P}, \preceq_{\mathcal{P}}\right)$ covers $\left(\mathcal{Q}, \preceq_{\mathcal{Q}}\right)$. Furthermore, since $\left(\mathcal{Q}, \preceq_{\mathcal{Q}}\right) \leq\left(\mathcal{P}, \preceq_{\mathcal{P}}\right)$ and $\mathcal{Q}$ is obtained form $\mathcal{P}$ by identifying the two related blocks $A$ and $B$, these blocks must form a convex set in $\left(\mathcal{P}, \preceq_{\mathcal{P}}\right)$, i.e., $A$ covers $B$ or conversely.

\section{FINITE POPARTITION LATTICES}

With the aid of Proposition 3.2, we are able to explicitly describe the lattices $\operatorname{PoPar}(X)$ where $X$ has 2 or 3 elements. Recall that when $X$ is finite, the lattice $\operatorname{Top}(X)$ of topologies on $X$ is isomorphic to $\operatorname{PoPar}(X)$. We will denote an $n$-element set $\{1,2, \ldots, n\}$ by $\underline{n}$. Let $S_{n, k}$ represent the number of partitions of $\underline{n}$ with $k$ blocks. These numbers, the Sterling numbers of the second kind, are discussed in Stanley [20]. Let $P_{k}$ denote the number of partial orders on $\underline{k}$. The numbers $P_{k}$ for $k=1,2, \ldots, 14$ are given in Erné and Stege [9]. It is easy to see that $|\operatorname{PoPar}(\underline{n})|=\sum_{k=1}^{n} S_{n, k} P_{k}$. The values of $|\operatorname{PoPar}(\underline{n})|$ are given for small $n$ in Table 4.1. The numbers $|\operatorname{PoPar}(\underline{n})|=|\operatorname{Top}(\underline{n})|$ tell how many topologies there are on an $n$-element set. For more values of the sequences given in 
Table 4.1, see Erné and Stege [9]. The number $|\operatorname{Par}(\underline{n})|$ of partitions of an $n$-element set is called the $n^{\text {th }}$ Bell number, and is given by $\sum_{r=1}^{n} \sum_{k=0}^{r}(-1)^{k}\left(\begin{array}{l}r \\ k\end{array}\right)(r-k)^{n} / r$ !. More information on Bell numbers can be found in Stanley [20].

\begin{tabular}{|c|c|c|c|c|}
\hline \hline$n$ & $P_{n}=|\operatorname{Po}(\underline{n})|$ & $|\operatorname{Par}(\underline{n})|$ & $|\operatorname{Top}(\underline{n})|=|\operatorname{PoPar}(\underline{n})|$ & $|\operatorname{ToPar}(\underline{n})|$ \\
\hline 1 & 1 & 1 & 1 & 1 \\
2 & 3 & 2 & 4 & 3 \\
3 & 19 & 5 & 29 & 13 \\
4 & 219 & 15 & 355 & 75 \\
5 & 4231 & 52 & 6952 & 541 \\
6 & 130023 & 203 & 209527 & 4683 \\
7 & 6129859 & 877 & 9535241 & 47293 \\
8 & 431723379 & 4140 & 642779354 & 545835 \\
9 & 44511042511 & 21147 & 63260289423 & 7087261 \\
10 & 6611065248783 & 115975 & 8977053873043 & 102247563 \\
\hline \hline
\end{tabular}

Table 4.1

In $\operatorname{Top}(\underline{2})=\operatorname{PoPar}(\underline{2})$, the discrete partition of $\underline{2}$ with discrete order is the largest element. The popartitions $(\{\{1\},\{2\}\},\{1\} \prec\{2\})$ and $(\{\{1\},\{2\}\},\{2\} \prec\{1\})$ are simultaneously atoms and coatoms. The indiscrete partition $\underline{2}$ is the least element. The lattice $\operatorname{PoPar}(\underline{2})$ is shown in Figure 4.2.

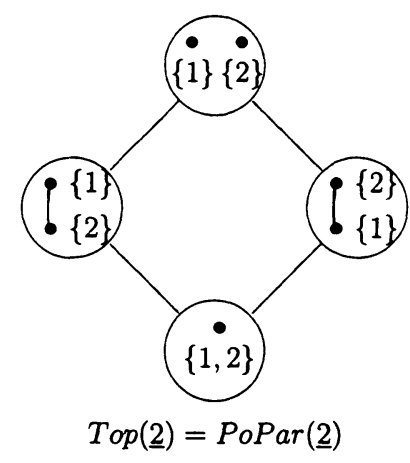

Figure 4.2

The 29 elements of $\operatorname{Top}(\underline{3})=\operatorname{PoPar}(\underline{3})$ are given numeric labels in Table 4.3 below. To avoid confusion with these labels, we will consider $\underline{3}$ to be the set $\{a, b, c\}$. The lattice $\operatorname{PoPar}(\underline{3})$ is shown in Figure 4.4. Notice that this diagram consists of a top element (labeled 1), a bottom element (labeled 29), three stacked crowns, $(\{2,3, \ldots, 13\},\{8,9, \ldots, 19\}$, and $\{14, \ldots, 19,23, \ldots, 28\})$ and three other elements $(20,21$, and 22). A nice three-dimensional model of this lattice diagram can be constructed by drawing the crowns in different colors on a clear cylinder and carefully placing the vertices 20,21 and 22 inside the cylinder, avoiding unnecessary intersections of lines (or points).

The coverings in $\operatorname{Top}(\underline{4})=\operatorname{PoPar}(\underline{4})$ are also easily obtained with the aid of Proposition 3.2. However, since PoPar( $\underline{4})$ has 355 elements, its Hasse diagram becomes unwieldly. The set $P o(\underline{4})$ of all partial orders on 4 elements appears at the upper end of PoPar( $\underline{4})$ as the popartitions of $\underline{4}$ whose underlying partitions are discrete. The Hasse diagram for $P o(\underline{4})$ has 219 vertices and 588 edges. We list 16 isomorphic classes of $P o(\underline{4})$ in Table 4.5, and indicate the covering relations 
among these classes in Figure 4.6. The weights on the edges of the graph in Figure 4.6 represent the number of elements from the larger isomorphism class that cover each element from the smaller isomorphism class. For example, the edge from III to VI has weight 3 , indicating that each partial order on $\underline{4}$ of type VI is covered by 3 partial orders of type III. These weights, together with the number of partial orders of each type (from Table 4.5) can be used to verify that the Hasse diagram for $\mathrm{Po}(\underline{4})$ has 588 edges.

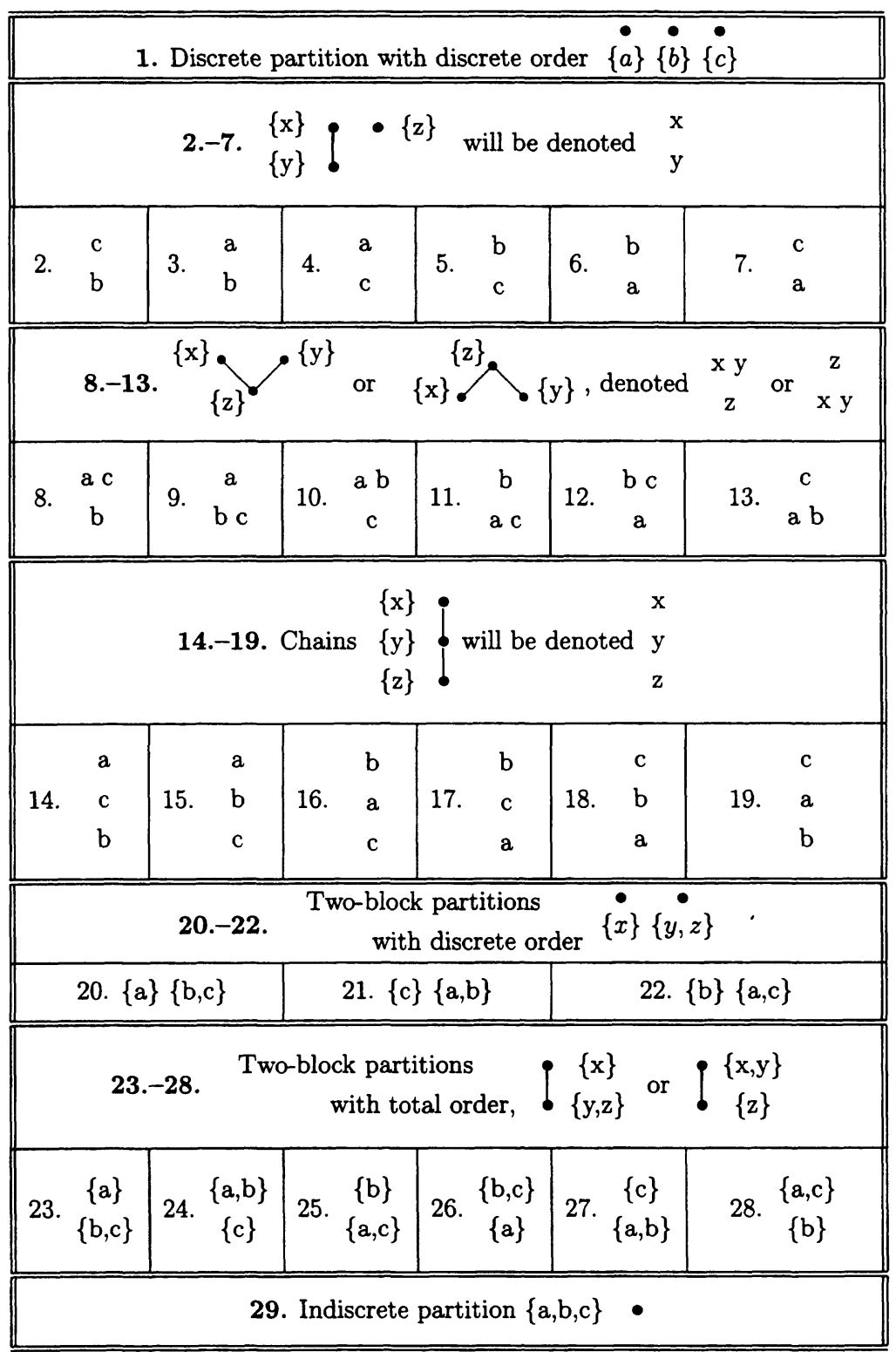

Elements of $\operatorname{Top}(\underline{3})=\operatorname{PoPar}(\underline{3})$

Table 4.3 


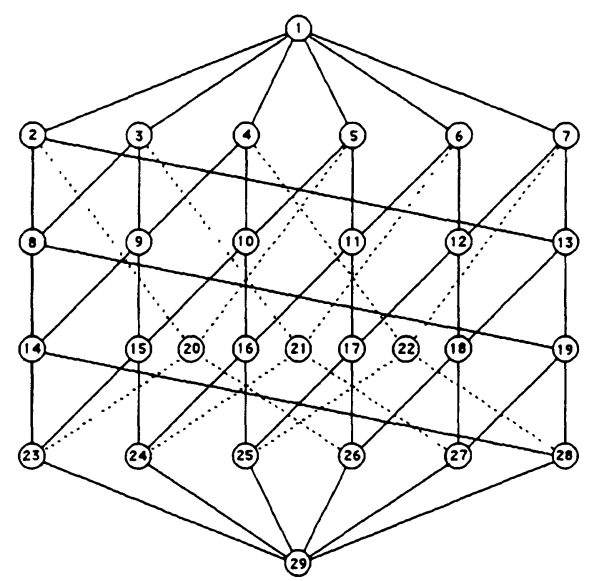

$\operatorname{Top}(\underline{3})=\operatorname{PoPar}(\underline{3})$

Figure 4.4

\begin{tabular}{|c|c|c|}
\hline $\begin{array}{c}\text { Isomorphism } \\
\text { Type }\end{array}$ & Diagram & $\begin{array}{c}\text { Number of p.o.s } \\
\text { of this type }\end{array}$ \\
\hline I & • & 1 \\
\hline II & - I. & 12 \\
\hline III & KK & 24 \\
\hline IV & & 12 \\
\hline V & & 24 \\
\hline VI & & 8 \\
\hline VII & & 24 \\
\hline VIII & 0 & 48 \\
\hline IX & & 6 \\
\hline $\mathrm{X}$ & & 12 \\
\hline XI & OR & 24 \\
\hline XII & & 24 \\
\hline
\end{tabular}

Types of Nonisomorphic Partial Orders on $\underline{4}$

Table 4.5 


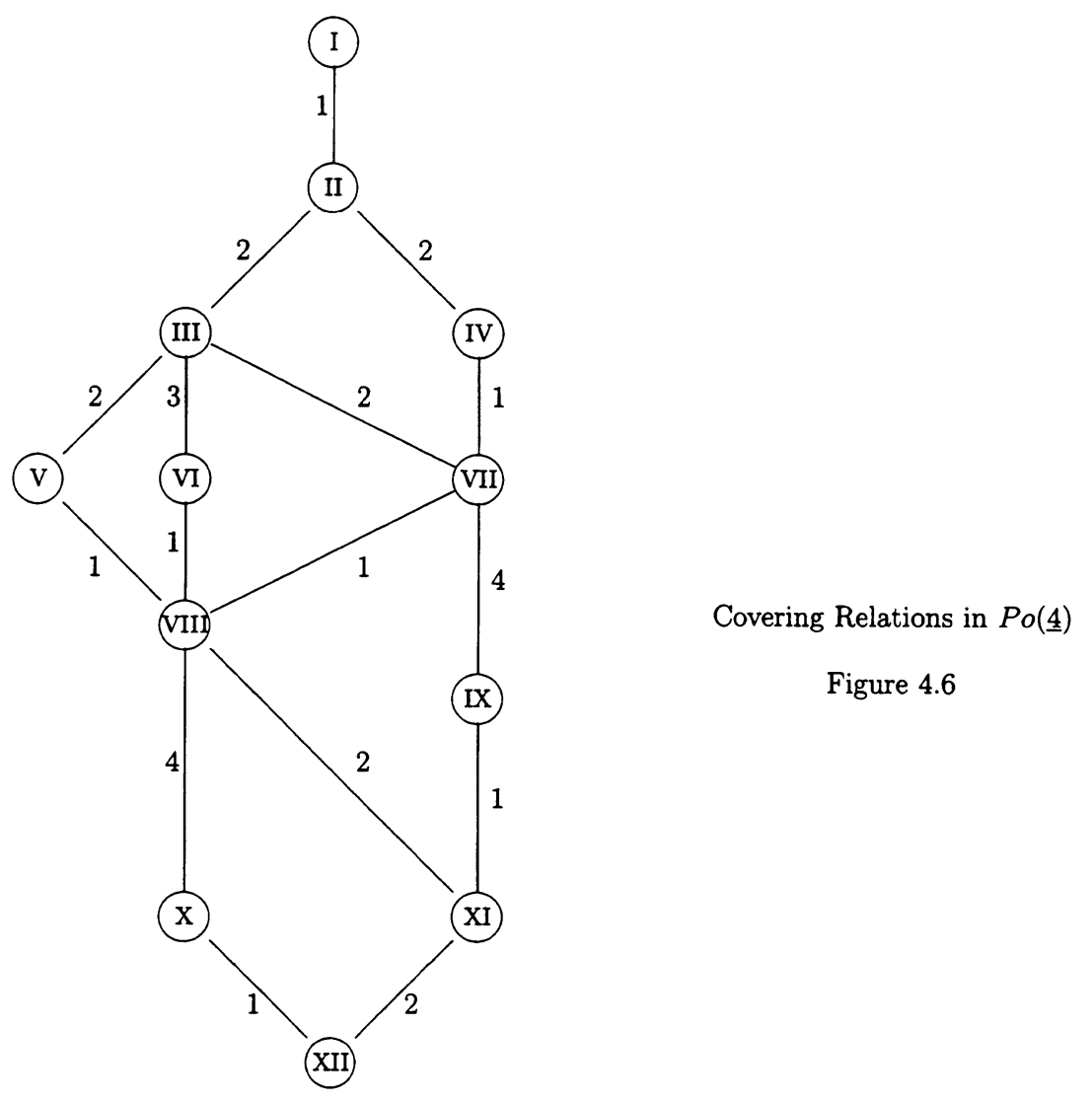

\section{TOTALLY ORDERED PARTITION LATTICES}

The set $\operatorname{ToPar}(X)$ of totally ordered partitions of $X$ is not a lattice if $|X|>1$, for if $|X|>1$, the totally ordered partitions $\left(\left\{A, A^{c}\right\}, A \prec A^{c}\right)$ and $\left(\left\{A, A^{c}\right\}, A^{c} \prec A\right)$ have no supremum. Arbitrary infima of members of $\operatorname{ToPar}(X)$ exist in $\operatorname{ToPar}(X)$ and agree with the infima in $\operatorname{PoPar}(X)$. To see this, it suffices to show that if $\left(\mathcal{P}_{\alpha}, \preceq_{\alpha}\right) \in \operatorname{ToPar}(X)$ for $\alpha \in A$, then $(\mathcal{P}, \preceq) \equiv \bigwedge_{\alpha \in A}\left(\mathcal{P}_{\alpha}, \preceq_{\alpha}\right)$ is totally ordered. Given $[a]_{\mathcal{P}},[b]_{\mathcal{P}} \in \mathcal{P},[a]$ and $[b]$ must be related in $\left(\mathcal{P}_{\alpha_{0}}, \preceq_{\alpha_{0}}\right)$ for each $\alpha_{0} \in A$ since $\left(\mathcal{P}_{\alpha_{0}}, \preceq_{\alpha_{0}}\right)$ is totally ordered. Thus, there is a chain of length 1 as in (2.1) between $[a]$ and $[b]$, so $[a]$ and $[b]$ are related in $(\mathcal{P}, \preceq)$. Thus, $\operatorname{ToPar}(X)$ is a $\wedge$-complete sublattice of $\operatorname{PoPar}(X)$.

The set $M$ of totally ordered discrete partitions of $X$ occupies a central position in $\operatorname{PoPar}(X)$. The elements of $M$ are the minimal elements of $\operatorname{Po}(X) \approx\left\{\left(\mathcal{P}, \preceq_{\mathcal{P}}\right) \in \operatorname{PoPar}(X): \mathcal{P}\right.$ is the discrete partition of $X\}$, and they are the maximal elements in $\operatorname{ToPar}(X)$. Indeed, in $\operatorname{PoPar}(X)$, $d(M) \cap \imath(M)=M, d(M) \approx \operatorname{ToPar}(X)$ and $i(M) \approx P o(X)$. This last fact says that for every partial order $\leq_{p}$ on $X$, there is a total order $\leq_{t}$ on $X$ such that id $:\left(X, \leq_{p}\right) \longrightarrow\left(X, \leq_{t}\right)$ is increasing; that is, every partial order on $X$ can be extended to a total order (see, e.g., Davey and Priestley [6], p.26). We also note that $d(M) \cap i(M)=M$ is the definition of $M$ being order convex. Equivalently, $M$ is order convex if $x \leq y \leq z$ and $x, z \in M$ implies $y \in M$.

If $X=\underline{n}$, it is easy to see that there are $n$ ! elements of $M$, corresponding to the permutations of $\underline{n}$. In PoPar( $(\underline{3})$ with the notation of Table 4.3 and Figure 4.4, the set $M$ of totally ordered discrete partitions is the set $\{14,15, \ldots, 19\}, \operatorname{Po}(\underline{3}) \approx i(M)=\{1,2, \ldots, 19\}, \operatorname{ToPar}(\underline{3}) \approx d(M)=$ 
$\{14, \ldots, 19,23, \ldots, 29\}$, and $i(M) \cup d(M)=\{1,2, \ldots, 29\} \backslash\{20,21,22\}$. The lattice diagram for the latter set can be obtained from Figure 4.4 by omitting the dashed lines and the three associated vertices.

For any totally ordered partition $\left(\mathcal{P}, \preceq_{\mathcal{P}}\right) \in \operatorname{ToPar}(X)$, the set $d(\mathcal{P})=\left\{\left(\mathcal{Q}, \preceq_{\mathcal{Q}}\right) \in \operatorname{PoPar}(X)\right.$ : $\left.\left(\mathcal{Q}, \preceq_{\mathcal{Q}}\right) \leq\left(\mathcal{P}, \preceq_{\mathcal{P}}\right)\right\}$ is a $\wedge$-complete sublattice of $\operatorname{PoPar}(X)$ with greatest element $\left(\mathcal{P}, \preceq_{\mathcal{P}}\right)$, and is therefore a complete lattice. The members of $d(\mathcal{P})$ are precisely the convex partitions of the totally ordered set $\left(\mathcal{P}, \preceq_{\mathcal{P}}\right)$. For example, if $\left(\mathcal{P}, \preceq_{\mathcal{P}}\right)$ is a totally ordered partition of $X$ order isomorphic to $\underline{n}$ with the natural order, then $d(\mathcal{P})$ corresponds to the convex partitions of $\underline{n}$. There are $2^{n-1}$ of these (there are twice as many convex partitions of $\underline{k}$ as of $\underline{k-1}$-you can add a new block $\{k\}$, or include $k$ in $[k-1])$. Thus, below each of the $n$ ! maximal elements in $\operatorname{ToPar}(\underline{n})$ are $2^{n-1}$ members of $\operatorname{ToPar}(\underline{n})$. Of course, one member of $\operatorname{ToPar}(\underline{n})$ may be below several maximal elements, so we may conclude $|\operatorname{ToPar}(\underline{n})| \leq n !\left(2^{n-1}\right)$. Recalling that $S_{n, k}$ gives the number of partitions of $\underline{n}$ with $k$ blocks and that there are $k$ ! total orders on $\underline{k}$, it is easy to see that $|\operatorname{ToPar}(\underline{n})|=\sum_{k=1}^{n}\left(S_{n, k}\right)(k !)$. The values of $|\operatorname{ToPar}(\underline{n})|$ for small values of $n$ are given in Table 4.1.

We may also generate the numbers $|\operatorname{ToPar}(\underline{n})|$ recursively. In an arbitrary totally ordered partition of $\underline{n}$, suppose the top block contains $k$ elements of $\underline{n}$. There are $\left(\begin{array}{l}n \\ k\end{array}\right)$ ways to choose these $k$ elements. The remaining $n-k$ elements can be formed into totally ordered partitions in $|\operatorname{ToPar}(\underline{n-k})|$ ways. Letting $k$ range from 1 to $n$, we have $|\operatorname{ToPar}(\underline{n})|=\sum_{k=1}^{n}\left(\begin{array}{l}n \\ k\end{array}\right)|\operatorname{ToPar}(\underline{n-k})|$. Here we take $|\operatorname{ToPar}(\underline{0})|$ to be 1 .

A chain topology on $X$ is a topology on $X$ whose open sets are totally ordered by inclusion. Chain topologies are considered in Doyle [7] and Stephen [23]. The proposition below may be used to translate the recursive formula for $|\operatorname{ToPar}(\underline{n})|$ in the preceding paragraph into the recursive formula given by Stephen [23, Theorem 2] for counting the number of chain topologies on $\underline{n}$.

PROPOSITION 5.1. A popartition $(\mathcal{P}, \preceq)$ of $X$ is a totally ordered partition if and only if the specialization topology $\tau_{\mathcal{P}}$ is a chain topology on $X$.

PROOF. For $x \in X$, let $N(x)=\bigcap\left\{N \in \tau_{\mathcal{P}}: x \in N\right\}$. The result follows easily from the equivalence of these three statements: (1) $N(x) \subseteq N(y)$, (2) $y \in c l\{x\}$, (3) $y \leq x$.

Doyle [7] defines a tower space on $n$ points to be any space homeomorphic to $\underline{n}$ with the chain topology $\{\emptyset, \underline{1}, \underline{2}, \underline{3}, \ldots, \underline{n}\}$. More generally, we will say a chain topology' $\tau$ on $X$ is a tower topology on $X$ if for every $U \in \tau$ there exists $V \in \tau$ and $x \in X \backslash V$ with $V \cup\{x\}=U$. Doyle shows that a finite topological space $X$ is a $\mathrm{T}_{0}$-space if and only if there is a continuous one-to-one function from $X$ to a tower space. We will show that this is equivalent to the statement that a quasiorder on a finite set $X$ is a partial order if and only if it can be extended to a total order on $X$. First, we will give a well known fundamental result on functions.

PROPOSITION 5.2. If $\left(X, \leq_{X}\right)$ and $\left(Y, \leq_{Y}\right)$ are quasiordered sets with associated specialization topologies $\tau_{X}$ and $\tau_{Y}$, respectively, then $f:\left(X, \leq_{X}\right) \longrightarrow\left(Y, \leq_{Y}\right)$ is increasing if and only if $f:\left(X, \tau_{X}\right) \longrightarrow\left(Y, \tau_{Y}\right)$ is continuous.

PROOF. Suppose $f:\left(X, \leq_{X}\right) \longrightarrow\left(Y, \leq_{Y}\right)$ is increasing. If $U$ is open in $\left(Y, \tau_{Y}\right)$, then $U$ is decreasing in $\left(Y, \leq_{Y}\right)$, and it follows that $f^{-1}(U)$ is decreasing in $\left(X, \leq_{X}\right)$, i.e., $f^{-1}(U)$ is $\tau_{X^{-}}$ open. Conversely, suppose $f:\left(X, \tau_{X}\right) \longrightarrow\left(Y, \tau_{Y}\right)$ is continuous. Then, from the definition of the topologies involved, the inverse image of any $\leq_{Y}$-decreasing set is $\leq_{X}$-decreasing. Suppose $a \leq_{X} b$ yet $f(a) \mathbb{Z}_{Y} f(b)$. Then $f(a) \notin d_{Y}(f(b))$. But since $f^{-1}\left(d_{Y}(f(b))\right)$ is a $\leq_{X}$-decreasing set that contains $b$, we have $a \in f^{-1}\left(d_{Y}(f(b))\right)$, contrary to $f(a) \notin d_{Y}(f(b))$. 
Recalling that the specialization order for a topology is a partial order if and only if the topology is $T_{0}$, we now observe that the following statements are equivalent:

(a) a quasiorder on $\underline{n}$ is a partial order if and only if it can be extended to a total order on $\underline{n}$.

(b) a quasiorder $\leq$ on $\underline{n}$ is a partial order if and only if there is a total order $\leq_{t}$ on $\underline{n}$ such that $i d:(\underline{n}, \leq) \longrightarrow\left(\underline{n}, \leq_{t}\right)$ is increasing.

(c) A topology $\tau$ on $\underline{n}$ is $\mathrm{T}_{0}$ if and only if there is a total order $\leq_{t}$ on $\underline{n}$ such that $i d:(\underline{n}, \tau) \longrightarrow$ $\left(\underline{n}, \tau_{\leq_{t}}\right)$ is continuous.

Noting that the specialization topology $\tau_{\leq t}$ from a total order on a finite set gives a tower space, and that the identity function is trivially one-to-one, we see that the following statement is implied by those above.

(d) A topology $\tau$ on $\underline{n}$ is $\mathrm{T}_{0}$ if and only if there is a one-to-one continuous function from $\underline{n}$ onto a tower space.

Since the function in (d) is a permutation on $\underline{n}$ and since any two tower spaces on $\underline{n}$ are homeomorphic, we may assume, without loss of generality, that the function in (d) is the identity. Furthermore, since any tower space topology on $\underline{n}$ is the specialization topology for some total order on $\underline{n}$, statement (d) implies (c). Thus, all of the statements (a)-(d) are equivalent.

The lattice $\operatorname{PoPar}(X)$ is isomorphic to the lattice $\operatorname{Pr} T o p(X)$ of principal topologies on $X$. By Proposition 5.1, $\operatorname{ToPar}(X)$ is isomorphic to the principal chain topologies on $X$. For the set $M$ of totally ordered discrete partitions of $X$, we have $M=i(M) \cap d(M) \approx P o(X) \cap \operatorname{ToPar}(X)$. Recalling that the lattice $Q(X)$ of quasiorders on $X$ is isomorphic to $\operatorname{Pr} T o p(X) \approx \operatorname{PoPar}(X)$ and that the partial orders on $X$ correspond to $T_{0}$ principal topologies, we see that $M \approx$ $\operatorname{Po}(X) \cap T o P a r(X) \approx\left\{T_{0}\right.$ principal topologies on $\left.X\right\} \cap\{$ principal chain topologies on $X\} \approx\{$ tower topologies on $X\}$. Thus, the set $T$ of tower topologies on $X$ occupies a central position in $\operatorname{PrTop}(X)$, with $i(T)$ isomorphic to the $\vee$-complete semilattice of $T_{0}$ principal topologies on $X$, and $d(T)$ isomorphic to the $\wedge$-complete semilattice of principal chain topologies on $X$. Again recall that if $X$ is finite, all topologies on $X$ are principal and we could omit the word "principal" in the discussion above.

\section{OTHER LATTICE PROPERTIES}

In this section, we will assume some familiarity with some standard lattice theory concepts as given in Birkhoff [4]. Background material on continuous lattices can be found in Gierz et al. [11].

$\operatorname{PoPar}(X)$ is complemented. Some complements of $(\mathcal{P}, \preceq) \in \operatorname{PoPar}(X)$ are just the discretely ordered complements of $\mathcal{P}$ in $\operatorname{Par}(X)$. Thus, for $(\mathcal{P}, \preceq) \in \operatorname{PoPar}(X)$, if we let $A$ be a complete set of equivalence class representatives from $\mathcal{P}$, then the discretely ordered partition $\{A\} \cup\{\{x\}$ : $x \in X \backslash A\}$ is a complement of $\mathcal{P}$. If $|X| \geq 3$, then there exist popartitions $(\mathcal{P}, \preceq)$ that are neither the top nor bottom element of $\operatorname{PoPar}(X)$ and have one block with more than one element of $X$. For such a popartition $\mathcal{P}$, the complete set of equivalence class representatives for $\mathcal{P}$ is not unique, that is, $\mathcal{P}$ is not uniquely complemented in $\operatorname{Par}(X)$. It follows that if $|X| \geq 3$, then the elements of $\operatorname{PoPar}(X)$ are not uniquely complemented. Since complements are unique in distributive lattices, we see that $\operatorname{Par}(X)$ and $\operatorname{PoPar}(X)$ are not distributive if $|X| \geq 3$. While any interval in $\operatorname{Par}(X)$ is complemented (Ore [18, p.596]), this result does not hold in $\operatorname{PoPar}(X)$, for, e.g., in $\operatorname{PoPar}(\underline{3})$ (see Figure 4.4), 27 has no complement in the interval $[29,13]$. 
In a complete lattice $L$, we say $x$ is way below $y$, denoted $x \ll y$ if for any directed subset $D \subseteq L, y \leq \sup D$ implies there exists $d \in D$ with $x \leq d$. A complete lattice $L$ is a continuous lattice if for every $x \in L, x=\sup \{y \in L: y \ll x\}$. In a finite lattice $x \ll y$ if and only if $x \leq y$ (See Remark 1.5, p.41 in Gierz et al. [11]), and thus every finite lattice is a continuous lattice. In particular, if $X$ is finite, $\operatorname{PoPar}(X)$ is a continuous lattice.

If $X$ is infinite, then $\operatorname{PoPar}(X)$ is not a continuous lattice. To see this, we will show that if $X$ is infinite, then $\mathcal{P} \ll \mathcal{Q}$ in $\operatorname{PoPar}(X)$ if and only if $\mathcal{P}$ is the bottom element of $\operatorname{PoPar}(X)$, i.e., the indiscrete (one-block) partition of $X$. (For simplicity, we will supress the orders on all popartitions in this paragraph.) Suppose $X$ is infinite. Embed a copy $\mathbf{N}$ of the natural numbers in $X$. For each $z \in X$ and each $n \in \mathrm{N}$, define $\mathcal{D}_{z}(n)$ (respectively, $\mathcal{D}^{z}(n)$ ) to be the discrete partition of $X$ with the order $\{z\} \leq\{m\}$ (respectively, $\{z\} \geq\{m\}$ ) for all $m \in \mathbf{N} \subseteq X$ with $m \geq n$. Define $\mathrm{D}_{z}=\left\{\mathcal{D}_{z}(n): n \in \mathrm{N}\right\}$ and $\mathrm{D}^{z}=\left\{\mathcal{D}^{z}(n): n \in \mathbf{N}\right\} . \mathrm{D}_{z}$ and $\mathrm{D}^{z}$ are directed sets in $\operatorname{PoPar}(X)$ with $\mathcal{D}_{z}(n) \leq \mathcal{D}_{z}(m)$ if and only if $\mathcal{D}^{z}(n) \leq \mathcal{D}^{z}(m)$ if and only if $n \leq m$. Thus, $\mathrm{D}^{z}$ and $\mathrm{D}_{z}$ are chains in $\operatorname{PoPar}(X)$ isomorphic to $\mathrm{N}$. Now for any $z \in X$, sup $\mathrm{D}_{z}=\sup \mathrm{D}^{z}$ is the top element in $\operatorname{PoPar}(X)$, namely, the discrete partition of $X$ with the discrete order. Thus, $\mathcal{Q} \leq \sup \mathrm{D}_{z}=\sup$ $\mathrm{D}^{z}$ for any $\mathcal{Q} \in \operatorname{PoPar}(X)$. For $\mathcal{P} \ll \mathcal{Q}$ to hold, we must have $\mathcal{P} \leq \mathcal{D}_{z}\left(n_{z}\right)$ and $\mathcal{P} \leq \mathcal{D}^{z}\left(m_{z}\right)$ for some $m_{z}, n_{z} \in \mathbf{N}$ and for every $z \in X$. For $z \in X$, define $T_{z}=\left\{j \in \mathbf{N}: j \geq m_{z}, j \geq n_{z}\right\}$. Now $\mathcal{P} \ll \mathcal{Q}$ implies $\mathcal{P}$ is a popartition with $\left[t_{z}\right]_{\mathcal{P}} \leq[z]_{\mathcal{P}} \leq\left[t_{z}\right]_{\mathcal{P}}$ for every $z \in X$ and every $t_{z} \in T_{z}$. Thus, in $\mathcal{P}$, every point $z$ of $X$ is identified with a tail $T_{z}$ of the sequence $\mathbf{N}$. Since all such tails overlap, $\mathcal{P}$ is the indiscrete partition of $X$. It follows that $\operatorname{PoPar}(X)$ is not continuous, since any nondiscrete popartition is not the supremum of popartitions way below it.

For any natural number $n, \operatorname{Par}(\underline{n})$ is a semimodular lattice. Since the Jordan-Dedekind chain condition (all maximal chains between two given points have the same length) holds in any semimodular poset of finite length, $\operatorname{Par}(\underline{n})$ is graded by its height function $h(\mathcal{P})=$ maximum length of a chain from the indiscrete partition to $\mathcal{P}$. (See pp. 5, 15-16, 40 of Birkhoff [4]). We will show that for $n>2, \operatorname{PoPar}(\underline{n})$ does not satisfy the Jordan-Dedekind chain condition, and therefore is neither semimodular nor graded. for $n>2$, consider popartitions $\mathcal{P}_{1}, \mathcal{P}_{2}, \mathcal{P}_{3}$ all with the discrete partition of $\underline{n}$, and with $1<2$ in $\mathcal{P}_{1}, 1<2,1<3$ in $\mathcal{P}_{2}$, and $1<2<3$ in $\mathcal{P}_{3}$, with no other order except $x \leq x$ for all $x \in \underline{n}$. Let $\mathcal{P}_{4}$ and $\mathcal{P}_{5}$ be popartitions whose underlying partition is $\{\{1,2\}\} \cup\{\{x\}: x=3,4, \ldots, n\}$, with $\mathcal{P}_{4}$ carrying the discrete order, and with $\{1,2\}<\{3\}$ in $\mathcal{P}_{5}$. Now $\mathcal{P}_{1}, \mathcal{P}_{2}, \mathcal{P}_{3}, \mathcal{P}_{5}$ and $\mathcal{P}_{1}, \mathcal{P}_{4}, \mathcal{P}_{5}$ are maximal chains from $\mathcal{P}_{1}$ to $\dot{\mathcal{P}}_{5}$ with different lengths.

ACKNOWLEDGMENTS. The author would like to extend his thanks to the General Algebra working group at Technische Hochschule Darmstadt for their kind hospitality during the preparation of this paper, and to Prof. Marcel Erné at Universität Hannover.

\section{References}

[1] AIGNER, M., Beiträge zum Verband der Partitionen, Habilitationsschrift im Fachbereich Mathematik, Eberhard-Karls-Universität, Tübingen, Germany, 1971.

[2] ALEXANDROFF, P., Diskrete Räume, Mat. Sb. (N.S.) 2 (1937) 501-518.

[3] ANDIMA, S. J. and THRON, W. J., Order-induced topological properties, Pacific J. Math. 75 (2) (1978) 297-318.

[4] BIRKHOFF, G., Lattice Theory, Third edition, American Mathematical Society, Providence, RI, 1967. 
[5] BROUGHAN, K. A., On the number of structures of reflexive and transitive relations, Canad. J. Math., 25, no.6 (1973) 1269-1273.

[6] DAVEY, B. A. and PRIESTlEY, H. A., Introduction to Lattices and Order, Cambridge University Press, New York, 1990.

[7] DOYLE, P. H., On finite $\mathrm{T}_{0^{-}}$-spaces, in General Topology and its Relation to Modern Analysis and Algebra, II (Proc. Second Prague Topological Sympos., 1966) pp. 115-117, Academia, Prague, 1967.

[8] ERNÉ, M., Struktur und Anzahlformeln für Topologien auf endlichen Mengen, Manuscripta Math., 11 (1974) 221-259.

[9] ERNÉ, M. and STEGE, K., Counting finite posets and topologies, Order, 8 (1991) 247-265.

[10] FRÖHLICH, O., Das Halbordnungssystem der topologischen Räume auf einer Menge, Math. Annalen, 156 (1964) 79-95.

[11] GIERZ, G., HOFMANN, K. H., KEIMEL, K., LAWSON, J. D., MISLOVE, M., and SCOTT, D. S., A Compendium of Continuous Lattices, Springer-Verlag, New York, 1980.

[12] HAWRYLYCYZ, M. and REINER, V., The lattice of closure relations on a poset, Algebra Universalis, 30 (1993) 301-310.

[13] KARIMPOUR, R. G., Complementary topology and Boolean rings, Tamkang Journal of Mathematics, 22 (1) (1991).

[14] KONG, T. Y., KOPPERMAN, R. D., and MEYER, P. R., A topological approach to digital topology, American Math. Monthly, 98 (1991) 901-917.

[15] KOPPERMAN, R. D., Asymmetry and duality in topology, preprint.

[16] KOWALSKY, H. J., Topological Spaces, Academic Press, New York, 1965.

[17] MARTIN, A. D. and ABIAN, A., Retrieving a topological space from its lattice of open sets, Publ. Math. Debrecen, 40/1-2 (1992) 11-16.

[18] ORE, O., Theory of equivalence relations, Duke Math. J., 9 (1942) 573-627.

[19] PUDLÁK, P. and TŮMA, J., Every finite lattice can be embedded in a finite partition lattice, Algebra Universalis, 10 (1980) 74-95.

[20] STANLEY, R. P., An Enumerative Combinatorics, Volume I, Wadsworth \& Brooks/Cole Advanced Books \& Software, Monterey, CA, 1986.

[21] STEEN, L. A. and SEEBACH, JR., J. A., Counterexamples in Topology, Second Edition, Springer Verlag, New York, 1978.

[22] STEINER, A. K., The lattice of topologies: Structure and complementation, Trans. Am. Math. Soc., 122 (1966) 379-398.

[23] STEPHEN, D., Topology on finite sets, American Math. Monthly, 75 (1968) 739-741.

[24] THRON, W. J., Lattice-equivalence of topological spaces, Duke Math. J., 29 (4) (1962) 671-679. Topological Structures, Holt, Rinehart and Winston, New York, 1966.

[26] THRON, W. J. and ZIMMERMAN, S. J., A characterization of order topologies by means of minimal $\mathrm{T}_{0}$-topologies, Proc. Am. Math.Soc, 27 (1) (1971) 161-167.

[27] TŮMA, J., On the structure of quasi-ordering lattices, preprint.

[28] VAIDYAnAThaSWAMY, R., Set Topology, Second Edition, Chelsea Publishing Co., New York, 1960.

[29] WARREN, R. H., The number of topologies, Houston J. Math. 8 (2) (1982) 297-301. 


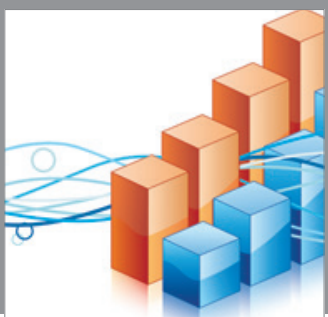

Advances in

Operations Research

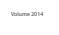

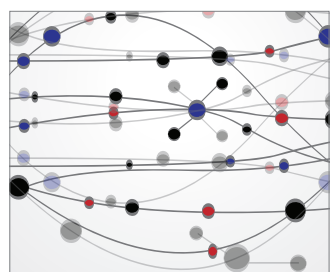

\section{The Scientific} World Journal
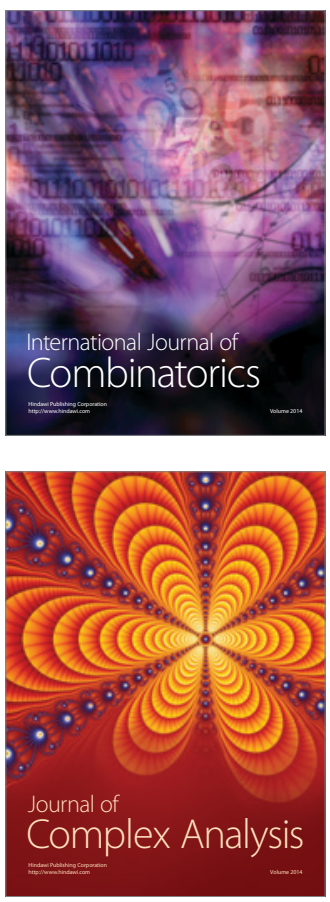

International Journal of

Mathematics and

Mathematical

Sciences
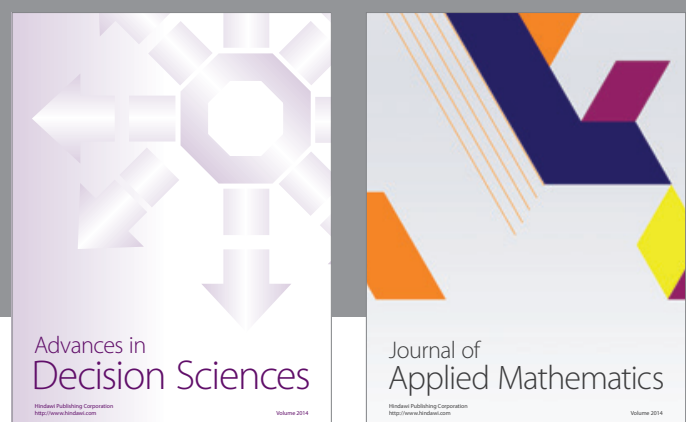

Journal of

Applied Mathematics
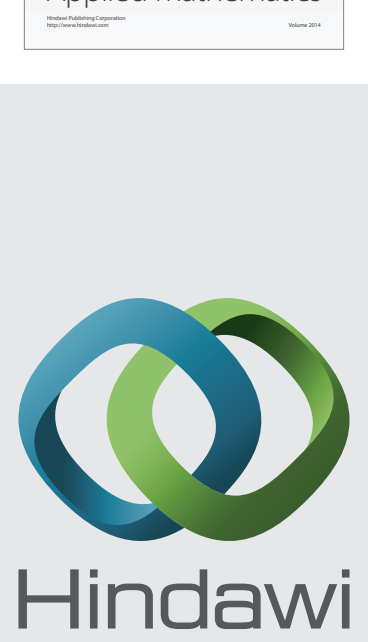

Submit your manuscripts at http://www.hindawi.com
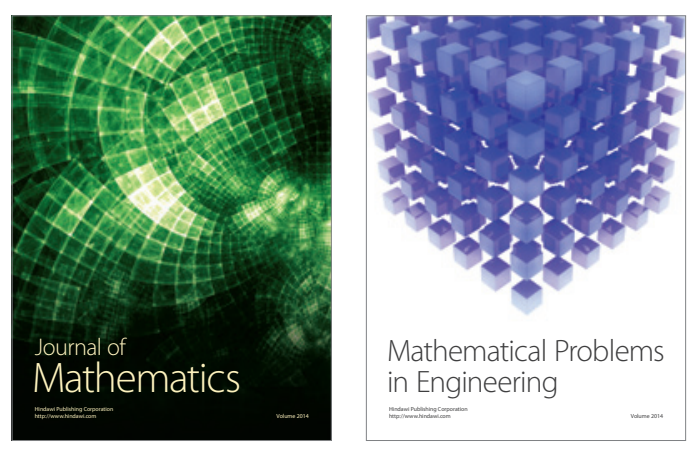

Mathematical Problems in Engineering
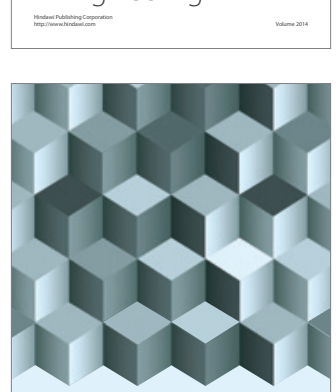

Journal of

Function Spaces
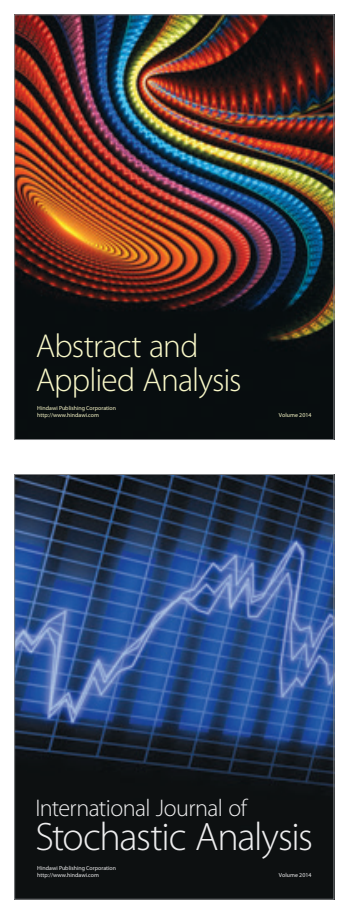

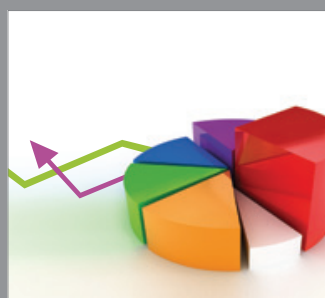

ournal of

Probability and Statistics

Promensencen
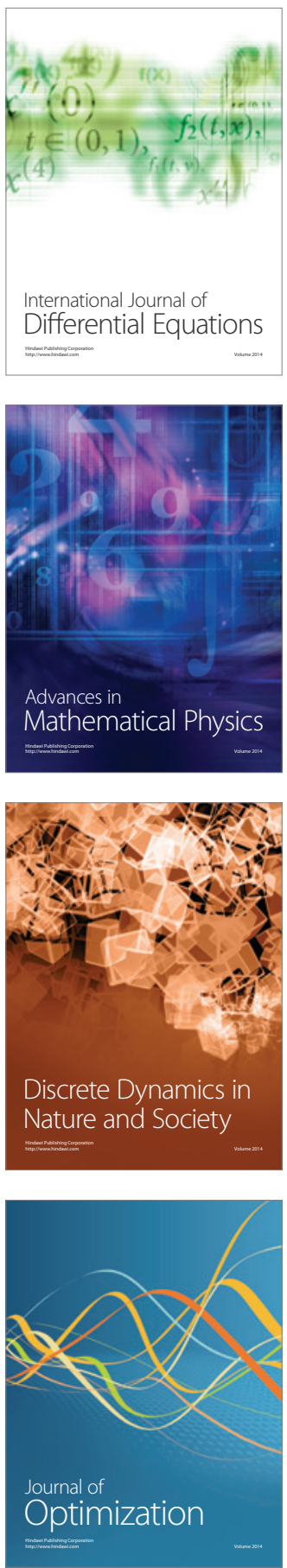